\title{
Possible Influence of Solar Activity on Global Volcanicity
}

\author{
Lihua $\mathrm{Ma}^{1}$, Zhiqiang Yin ${ }^{1} \&$ Yanben Han $^{1}$ \\ ${ }^{1}$ National Astronomical Observatories, Chinese Academy of Sciences, Beijing, China \\ Correspondence: Zhiqiang Yin, National Astronomical Observatories, Chinese Academy of Sciences, Beijing \\ 100012, China. Tel: 86-10-64860253. E-mail: yinzhq@nao.cas.cn
}

Received: January 4, 2018

Accepted: January 16, 2018

Online Published: January 23, 2018

doi:10.5539/esr.v7n1p110

URL: https://doi.org/10.5539/esr.v7n1p110

The research is financed by a grant from National Basic Research Program of China (2012CB957801).

\begin{abstract}
Solar activity has been found to be strongly correlated with some geophysical processes on the Earth system. In the present paper, using global dust veil index of volcanic eruptions and sunspot numbers indicating solar activity, the authors investigate the possible influence of solar activity on global volcanicity during 1700-1995. Results of wavelet analysis indicate that variations of global volcanicity have remarkable temporal characteristics, and it is high related to solar activity on about 11 -year cycle, with exceeding the $5 \%$ statistical significance level against red noise.
\end{abstract}

Keywords: global volcanicity, solar activity, wavelet transform

\section{Introduction}

The Earth system is dominated by electric-magnetic radiation, partial radiation, and solar cosmic rays from the Sun. Any subtle variations of solar activity are manifested in geophysical parameters including meteorologic, oceanic, Earth's inner. Some studies show solar activity relates to geophysical processes (including some natural hazards phenomena, such as earthquake, volcanic eruption and magnetic storm) to some extent. A statistically significant connection between solar activity and geomagnetic activity was found for the reason of influence of solar electric-magnetic radiation (Alania et al., 2004). Magnetic storms during maximum years of solar activity may affect property of faults and gestate great earthquakes in some region (Han et al., 2004). Love and Thomas (2013) studied statistically solar-terrestrial interaction between earthquakes and solar activity. When the Earth's inner energy is accumulated to certain extent, hot magma under huge inner press squirts from the Earth's inner along the weak region of the crust and forms volcano. Every volcanic eruption will emit a lot of lava, tephra and gases. This brings out many environmental problems, including greenhouse effect, ozone hole and acid rain. Some studies showed there is close relationship among volcanic eruption, earthquake, solar activity and climatic change (Schneider et al., 1975; Kelly et al., 1996; Mann et al., 1998; Stenni et al., 2002). Mazzarella and Palumbo (1989) analyzed the 11-year modulation of worldwide large volcanic and seismic activity. Using the historical record of large volcanic eruptions, the relationship between volcanic activity and solar cycle were found (Stothers, 1989; Casati, 2014). In this work, the authors apply wavelet technique to investigate possible connection between global volcanicity and solar activity.

\section{Data Description}

\subsection{Dust Veil Index}

Volcanicity can be described with many different indices from various observations. Dust veil index (DVI) developed by Lamb is a quantitative method for comparing the magnitude of volcanic eruptions. The DVI value for any volcanic eruption is based on a review of the observational, empirical, and theoretical studies of the possible impact on climate of volcanic dust veils. The index is a numerical index that quantifies the impact of a particular volcanic eruption's release of dust and aerosols over years following the event, especially the impact on the Earth's energy balance. The DVI allows one to compare volcanic eruptions by a single numerical index, and it is an important data series of analysing global volcanicity. The weighted volcanic dust index series in this work extracted from National Climatic Data Center (NCDC) spans 1500 to 1995, at yearly intervals (Mann et al., 2000). The DVI series is plotted in Figure 1(a). 


\subsection{Sunspot Numbers}

A sunspot is a region on the Sun's photosphere that is marked by a lower temperature than its surroundings, and intense magnetic activity. The number of sunspots correlates with the intensity of solar radiation. In the middle of 19th century, Wolf devised a daily method of estimating solar activity by counting the number of individual spots and groups of spots on the face of the Sun. Now, Wolf sunspot counts continue and become an important data set of analysing solar activity. The yearly Wolf relative sunspot numbers (SN) data series is obtained from the sunspot index data center (SIDC) of Royal observatory of Belgium. The data set covers the period of time from 1700 to 2006. The SN series is plotted in Figure 1(b).
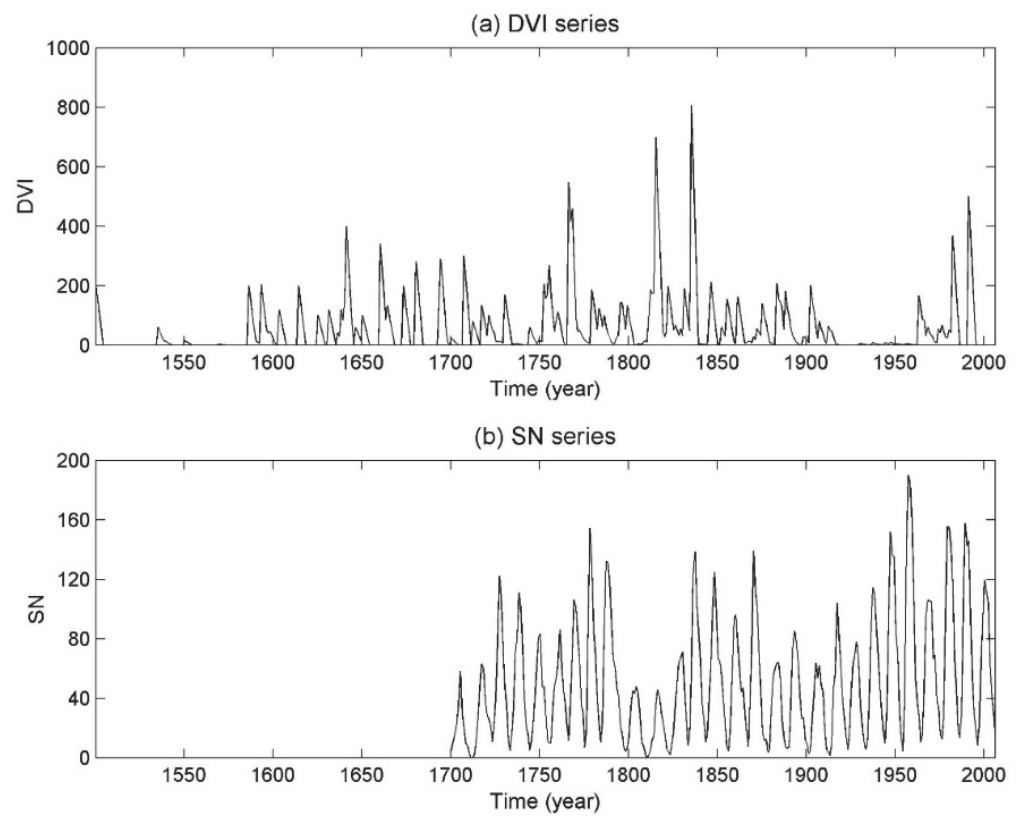

Figure 1. Subfigures (a) and (b) are corresponding to DVI series during 1500-1995 and SN series during 1700-2006, respectively

\section{Analysis and Comparison}

Wavelet technique is becoming a common tool for analyzing localized variations of power within a time series. By decomposing a time series into time-frequency space, one is able to determine both the dominant modes of variability and how those modes vary in time. The wavelet transform has been used for numerous studies in geophysics (for a review, see, e.g., Daubechies, 1992; Foufoula-Georgiou and Kumar, 1995, 1997; Torrence and Compo, 1998).

\subsection{Continuous Wavelet Transform}

The continuous wavelet transform (CWT) is used in this work. Morlet wavelet, as mother wavelet, is defined as,

$$
\psi_{0}(\eta)=\pi^{-1 / 4} e^{i \omega_{0} \eta} e^{-\eta^{2} / 2}
$$

Where $\omega_{0}$ is dimensionless frequency and $\eta$ is dimensionless time. The CWT of a time series with uniform time step $\delta t$, is defined as the convolution of $x_{n}$ with the scaled and normalized wavelet. Corresponding wavelet transform is as follows.

$$
W_{n}^{X}(s)=\sqrt{\frac{\delta t}{s}} \sum_{n^{\prime}=1}^{N} x_{n^{\prime}} \psi_{0}\left[\left(n^{\prime}-n\right) \frac{\delta t}{s}\right]
$$

We define the wavelet power as $\left|\mathrm{W}_{\mathrm{n}}^{\mathrm{x}}(\mathrm{s})\right|^{2}$. Considering edge-effect of the CWT, a Core of Influence (COI) is introduced. Here we take the COI as the area in which the wavelet power caused by a discontinuity at the edge has dropped to $e^{-2}$ of the value at the edge (Grinsted, et al., 2004). 
The statistical significance of wavelet power can be assessed relative to the null hypotheses that the signal is generated by a stationary process with a given background power spectrum $\left(P_{k}\right)$. Distinctive red noise characteristics in geophysical time series can be modeled very well by a first order autoregressive $\left(\mathrm{AR}_{1}\right)$ process. The Fourier power spectrum of an $\mathrm{AR}_{1}$ process with lag-1 autocorrelation $\alpha$ is given by

$$
P_{k}=\frac{1-\alpha^{2}}{\left|1-\alpha e^{-2 i \pi k}\right|^{2}}
$$

where $k$ is the Fourier frequency index (Allen and Smith, 1996). With Monte Carlo methods, the probability that the wavelet power with being greater than $p$ is

$$
D\left(\frac{\left|W_{n}^{X}(s)\right|^{2}}{\sigma_{X}{ }^{2}}<p\right)=\frac{1}{2} P_{k} \chi_{v}^{2}(p)
$$

Where, $P_{k}$ is a given power spectrum, and $v$ is equal to 1 for real and 2 for complex wavelets (Torrence and Compo, 1998).

Many statistical tests assume that the probability density function is close to normal. We standardize (zero mean, unit standard deviation) both time series and will refer to the standardized versions as simply DVI and SN series before wavelet transform.

The CWT of the DVI is shown on upper panel of Figure 2. Generally, there are a few situations where the coherence time is independent of the frequency in the wavelet power of the DVI time series. There are relatively obvious fluctuations features with significant peaks on 2 - 16-year time scales, about 10 years, and about 20 years with above the $5 \%$ significance level, and time span of corresponding time series is very short. Meanwhile, $\sim 30$ - and 60-year periodic fluctuations also exist in global volcanicity, though corresponding significance level is below the 5\%. The CWT of the SN is shown on lower panel of Figure 2. Obviously, remarkable oscillation in solar activity is about 11 -year cycle, with exceeding the $5 \%$ statistical significance level against red noise during the whole time span. About 22 years and 60 years may be oscillations of solar activity. It is hard to diagnose if possible connection exists in volcanicity and solar activity from Figure 2(a) and Figure 2(b). The cross wavelet transform helps in this regard.
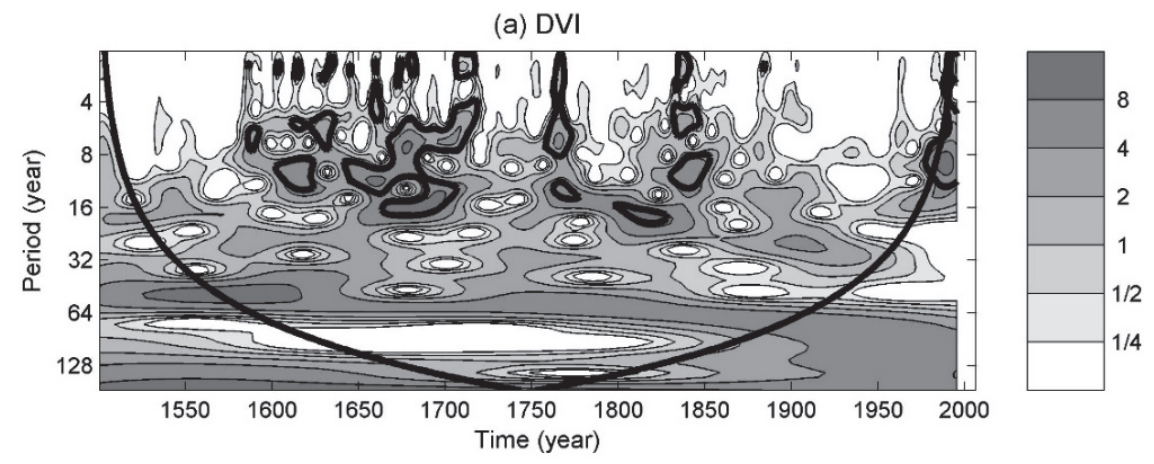

(b) $\mathrm{SN}$

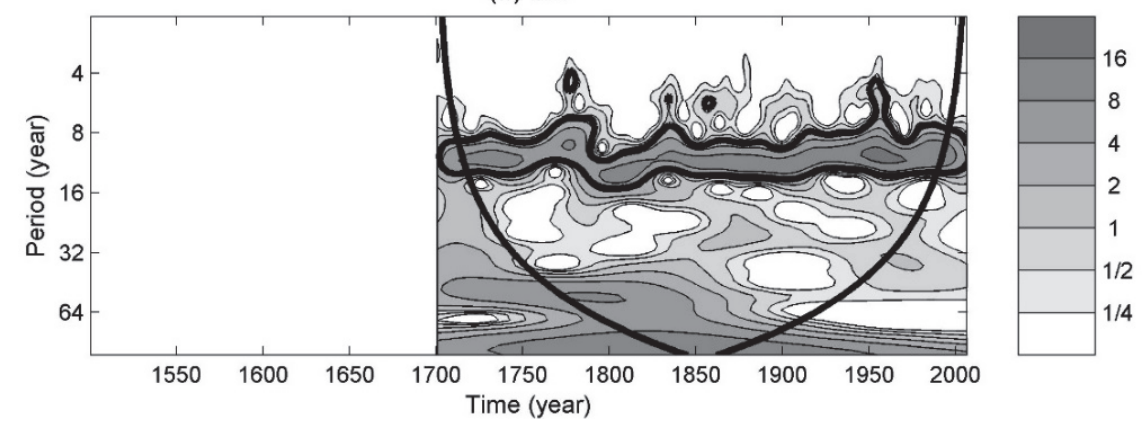

Figure 2. The continuous wavelet power spectrum of DVI (a) and SN (b) time series. The thick black contour designates the $5 \%$ significance level against red noise and the cone of influence (COI) where edge effects might distort the picture is shown as a lighter shade 


\subsection{Cross Wavelet Transform}

The cross wavelet transform (XWT) of two series $x_{n}$ and $y_{n}$ is defined as $W^{X Y}=W^{X} W^{Y^{*}}$, and the cross wavelet power is defined as $\left|W^{X Y}\right|$. Where, ${ }^{*}$ denotes complex conjugation. The complex $\operatorname{argument} \arg \left(W^{X Y}\right)$ can be interpreted as the local relative phase between $x_{n}$ and $y_{n}$ in time frequency space. If two time series with background power spectra are $P_{k}^{X}$ and $P_{k}^{Y}$ respectively, the theoretical distribution of the cross wavelet power of them is given as

$$
D\left(\frac{\left|W_{n}^{X}(s) W_{n}^{Y^{*}}(s)\right|^{2}}{\sigma_{X} \sigma_{Y}}<p\right)=\frac{Z_{v}(p)}{v} \sqrt{P_{k}^{X} P_{k}^{Y}}
$$

where $Z_{v}(p)$ is the confidence level associated with the probability $p$ for a probability density function defined by the square root of the product of two $\chi^{2}$ distributions (Torrence and Compo, 1998).

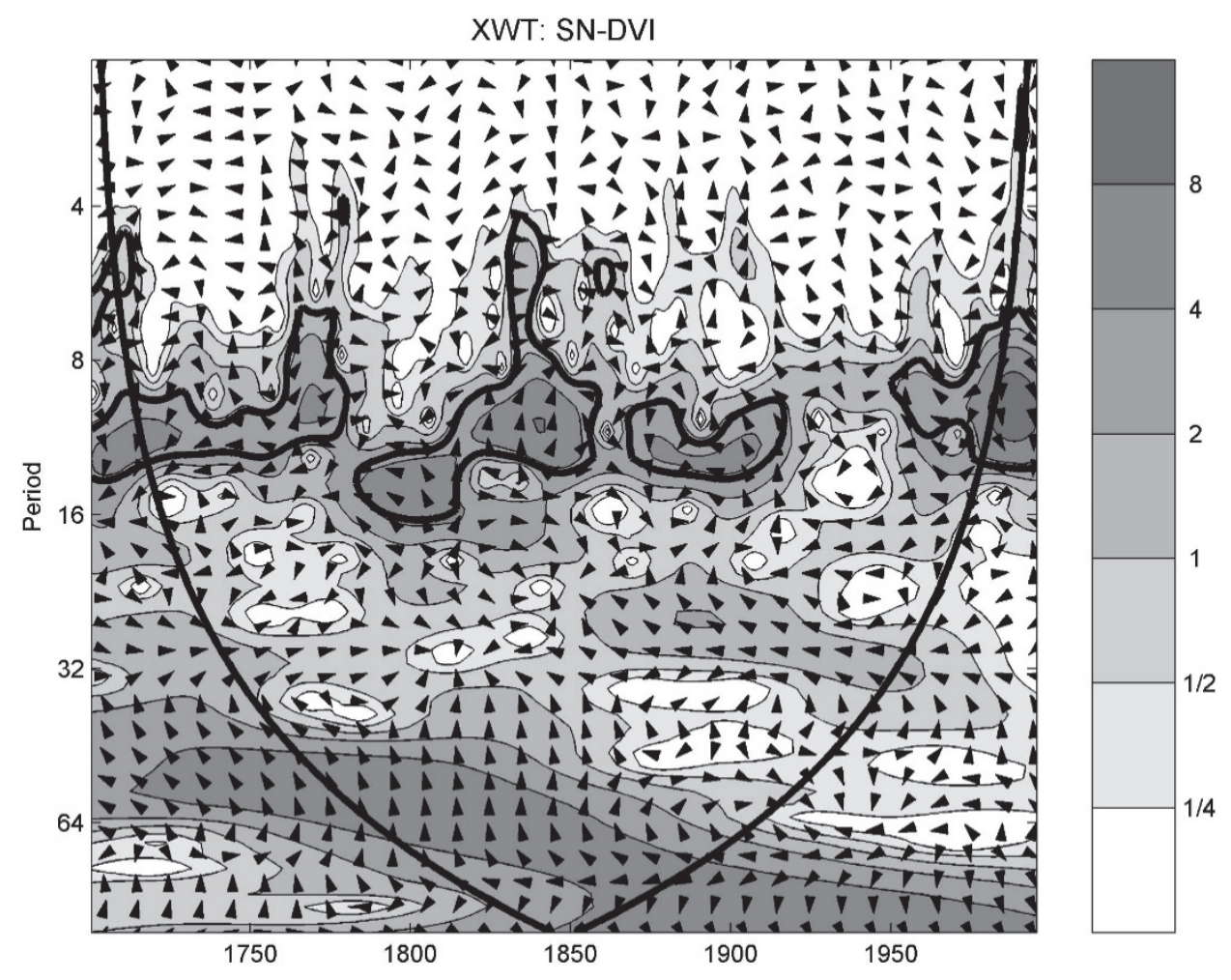

Figure 3. Cross wavelet transform of the standardized SN and DVI series during 1700-1995. The 5\% significance level against red noise is shown as a thick contour. The relative phase relationship is shown as arrows (with in-phase pointing right, anti-phase pointing left, and SN leading DVI by $90^{\circ}$ pointing straight up)

The XWT of global volcanicity and sunspot numbers is shown in Figure 3. Here, we notice that the common features we cannot find by eye from the individual wavelet transforms. Remarkable association signals exist on about 11-year solar cycle with above the 5\% significance level. Meanwhile, obvious connection on about 32-year and about 60-year time scales exist in the time span during $1850-1950$ and during $1700-1900$, respectively. We therefore speculate that there is a stronger link between global volcanicity and solar activity that implied by the cross wavelet power.

\subsection{Cross Wavelet Phase Angle}

In order to calculate the phase difference between the two time series, the mean and confidence interval of the phase over regions with higher than $5 \%$ statistical significance is estimated. Generally, calculating the mean phase is useful. The circular mean of a set of angles $\left(a_{i}, i=1, \ldots, n\right)$ is defined as (Zar, 1999) 


$$
\alpha_{m}=\arg (X, Y), \text { with } X=\sum_{i=1}^{n} \cos \left(a_{i}\right) \text { and } Y=\sum_{i=1}^{n} \sin \left(a_{i}\right)
$$

It is difficult to calculate the confidence interval of the mean angle reliably for the phase angles are not independent. Because the circular standard deviation is analogous to the linear standard deviation in that it varies from zero to infinity, and it can give similar results to the linear standard deviation when the angles are distributed closely around the mean angle. Therefore, we define the circular standard deviation as

$$
s=\sqrt{-2 \ln (R / n)}
$$

where $R=\sqrt{X^{2}+Y^{2}}$. In some cases there might be reasons for calculating the mean phase angle for each scale, and then the phase angle can be quantified as a number of years (Grinsted,et al., 2004). According to cross wavelet phase angle from Figure 3, we can find complicated phase relations between the SN series and the DVI series. Generally, the solar activity leads global volcanicity on about 11-year, about 32-year and about 60 -year time scales.

\subsection{Wavelet Coherence}

Cross wavelet power reveals areas with high common power. Another useful measure is how coherent the cross wavelet transform is in time frequency space. Following the work of Torrence and Webster (1998) the wavelet coherence of SN and DVI time series is calculated and the squared wavelet coherence of two time series is shown in Figure 4. All significant sections show SN leading DVI series. Meanwhile compared with the XWT the information about SN leading DVI is also shown in wavelet coherence spectrum with smaller area.

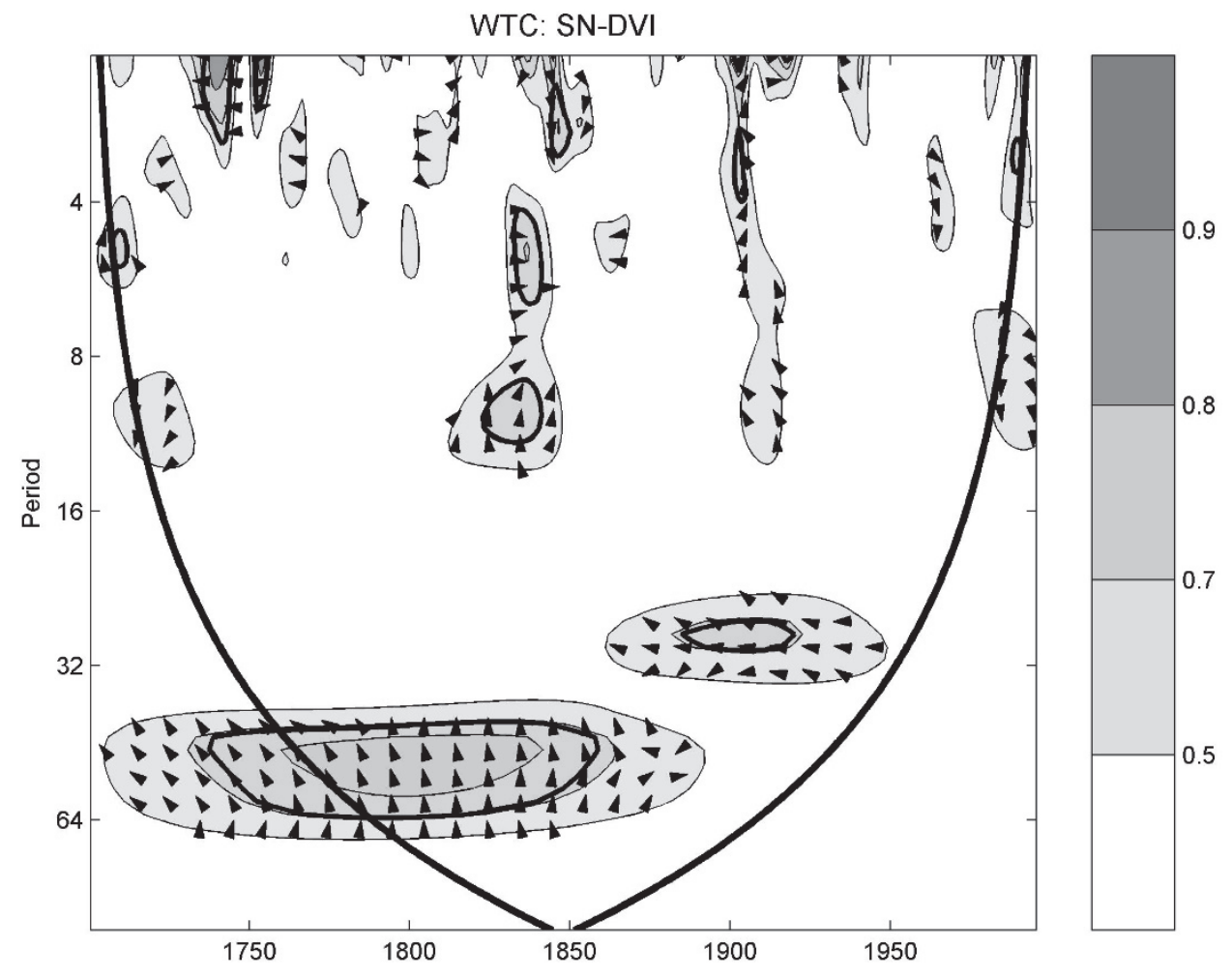

Figure 4. Squared wavelet coherence between the standardized SN and DVI time series. The $5 \%$ significance level against red noise is shown as a thick contour.

\section{Summary}

Solar ray radiation and particle radiation possibly effect geological structure of the Earth system and arouse volcanic eruption, and the Sun makes contribution to global volcanicity through complex energy/particle transport process. According to continuous wavelet transform, there are obvious periodic fluctuations with intermittent time in global volcanic activity. Remarkable connections are found from cross wavelet transform 
and wavelet coherence between global volcanicity and solar activity on about 11-year time scales, with exceeding the $5 \%$ statistical significance level. Meanwhile obvious evidence of solar activity leading global volcanicity can be found.

Actually, the establishment of a volcano is affected by some excitation sources from the exterior and interior of the solid Earth, such as change in topography, atmosphere, oceans, and mantle convection, etc, contributing their energies to excite it. Besides solar activity, there should possibly be many factors trigger volcanic eruption, still. The analysis of other factors influencing volcano will be included in an extended article in the future.

\section{Acknowledgements}

The authors are grateful to NCDC for providing weighted volcanic dust veil data series, to SIDC team for supporting sunspot numbers series (online catalogue of the sunspot index, http://sidc.oma.be/html/sunspot.html). Wavelet software is provided by C. Torrence and G. Compo, and is available at: http://paos.colorado.edu/research/wavelets. Software by Grinsted, A., et al., is available at https://github.com/grinsted/ wavelet-coherence.

\section{References}

Alania M. V., Gil, A., \& Modzelewska, R. (2004). On statistical relationship of solar, geomagnetic and human activities. Advances in Space Research, 34, 1602-1606. https://doi.org/10.1016/j.asr.2004.02.012

Allen, M. R., \& Smith, L. A. (1996). Monte Carlo SSA: Detecting irregular oscillations in the presence of $\begin{array}{llllll}\text { coloured } & \text { noise. } & J & \text { Clim. } & 9, & 3373-3404 .\end{array}$ https://doi.org/10.1175/1520-0442(1996)009<3373:MCSDIO>2.0.CO;2

Casati, M. (2014). Significant statistical relationship between great volcanic eruptions and the count of sunspots from 1610 to the present, NCGT Journal, 2, 78-81

Daubechies, I. (1992). Ten lectures on wavelets. Philadelphia: SIAM, 357.

Foufoula-Georgiou, E., \& Kumar, P. (1994). Wavelets in Geophysics, Academic Press, San Diego, USA, 373p.

Grinsted, A., Moore, J. C., \& Jevrejeva, S. (2004). Application of the cross wavelet transform and wavelet coherence to geophysical time series. Nonlinear Processes in Geophysics, 11, 561-566.

Han, Y. B., Guo, Z. J., Zhao, J., Wu, J. B., \& Ma, L. H. (2004). Possible triggering of solar activity to big earthquakes (Ms $\geq 8)$ in faults with near west-east strike in China. Science in China. Science in China, 47, 173-181. https://doi.org/10.1360/03yw0103

Kelly, P. M, Jones, P. D., \& Pengqun, J. (1996). The spatial response of the climate system to explosive volcanic eruptions, International Journal of Climatology, $16, \quad 537-550$. https://doi.org/10.1002/(SICI)1097-0088(199605)16:5<537::AID-JOC23>3.0.CO;2-F

Kumar, P., \& Foufoula-Georgiou, E. (1997). Wavelet analysis for geophysical applications, Rev. Geophys., 35, 385-412. https://dx.doi.org/10.1029/97RG00427

Love, J. J., \& Thomas, J. N. (2013). Insignificant solar-terrestrial triggering of earthquakes, Geophys. Res. Lett., 40, 1165-1170. https://doi.org/10.1002/grl.50211

Mann, M. E., Bradley, R. S., \& Hughes, M. K. (1998). Global-scale temperature patterns and climate forcing. over the past six centuries, Nature, 392, 779-787. https://doi.org/10.1038/33859

Mann, M. E., Gille, E. P., Bradley, R. S., Hughes, M. K., Overpeck, J. T., Keimig, F. T., \& Gross, W. S. (2000). Global Temperature Patterns in Past Centuries: An Interactive Presentation, Earth Interactions, 4, 1-29. https://doi.org/10.1175/1087-3562(2000) 004<0001:GTPIPC >2.3.CO;2

Mazzarella, A., \& Palumbo, A. (1989). Does the solar cycle modulate seismic and volcanic activity? Journal of Volcanology and Geothermal Research, 39, 89-93. https://doi.org/10.1016/0377-0273(89)90023-1

Schneider, S. H., \& Mass, C. (1975). Volcanic dust, sunspots and temperature trends, Science, 190, 741-746. https://doi.org/10.1126/science.190.4216.741

Stenni, B., Proposito, M., Gragnani, R., Flora, O., Jouzel, J., Falourd, S., \& Frezzotti, M. (2002). Eight centuries of volcanic signal and climate change at Talos Dome (East Antarctica), J. Geoph. Res., D9, 107, 1-14. https://doi.org/10.1029/2000JD000317

Stothers, R. B. (1989). Volcanic Eruptions and Solar Activity. J. Geoph. Res., 94, B12, 17371-17381. https://doi.org/10.1029/JB094iB12p17371. 
Torrence, C., \& Compo, G. P. (1998). A practical guide to wavelet analysis. Bull. Amer. Meteor. Soc., 79, 61-78. https://doi.org/10.1175/1520-0477(1998)079<0061:APGTWA>2.0.CO;2

\section{Copyrights}

Copyright for this article is retained by the author(s), with first publication rights granted to the journal.

This is an open-access article distributed under the terms and conditions of the Creative Commons Attribution license (http://creativecommons.org/licenses/by/4.0/). 